\title{
Standardized assessment of global longitudinal and circumferential strain - a modality independent software approach
}

\author{
Johannes Riffel ${ }^{1 *}$, Marius G Keller ${ }^{1}$, Matthias Aurich¹, Yannick Sander ${ }^{1}$, Florian Andre ${ }^{1}$, Sorin Giusca', \\ Fabian aus dem Siepen ${ }^{1}$, Sebastian Seitz ${ }^{1}$, Christian Galuschky², Grigorios Korosoglou', Derliz Mereles', \\ Hugo Katus ${ }^{1}$, Sebastian Buss ${ }^{1}$
}

From 18th Annual SCMR Scientific Sessions

Nice, France. 4-7 February 2015

\section{Background}

Myocardial deformation measurement is superior to left ventricular ejection fraction in identifying early changes in myocardial contractility and prediction of cardiovascular outcome. The lack of standardization hinders its clinical implementation.

The aim of the study is to investigate a novel standardized deformation imaging approach based on the feature tracking algorithm for the assessment of global longitudinal (GLS) and global circumferential strain (GCS) in echocardiography and cardiac magnetic resonance imaging (CMR).

\section{Methods}

70 subjects undergoing CMR were consecutively investigated with echocardiography within a median time of 30 min. GLS and GCS were analyzed with a post processing software incorporating the same standardized algorithm for both modalities. Accordingly, global strain was defined as the relative shortening of the whole, uni-segmented endocardial contour and calculated according to the strain formula.

\section{Results}

Mean GLS values were $-16.2 \pm 5.3 \%$ and $-17.3 \pm 5.3 \%$ for echocardiography and CMR, respectively. GLS did not differ significantly between the two imaging modalities, which showed strong correlation $(\mathrm{r}=0.86)$, a small bias $(-1.1 \%)$ and narrow $95 \%$ limits of agreement (LOA, $\pm 5.4 \%$ ). Mean GCS values were $-17.9 \pm 6.3 \%$ and -24.4 $\pm 7.8 \%$ for echocardiography and CMR, respectively. GCS was significantly underestimated by echocardiography $(\mathrm{p}<0.001)$. A weaker correlation $(\mathrm{r}=0.73)$, a higher bias $(-6.5 \%)$ and wider LOA $( \pm 10.5 \%)$ were observed for GCS. GLS showed a strong correlation $(r=0.92)$ when image quality was good, while correlation dropped to $\mathrm{r}=0.82$ with poor acoustic windows in echocardiography. GCS assessment revealed only a strong correlation $(r=0.87)$ when echocardiographic image quality was good. No significant differences for GLS between two different echocardiographic vendors could be detected.

\section{Conclusions}

Quantitative assessment of GLS using a standardized software algorithm allows the direct comparison of values acquired irrespective of the imaging modality. GLS may therefore serve as a reliable parameter for the assessment of global left ventricular function in clinical routine besides standard evaluation of the ejection fraction.

\section{Funding}

The study was supported by a grant from the B. Braun Stiftung. H.A.K. was supported by the DZHK (Deutsches Zentrum für Herz-Kreislauf-Forschung - German Centre for Cardiovascular Research).

\footnotetext{
Authors' details

${ }^{1}$ Cardiology, University of Heidelberg, Heidelberg, Germany. ${ }^{2}$ TomTec, Unterschleissheim, Germany.
} 


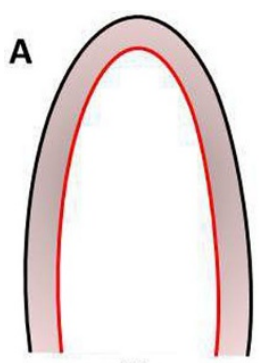

ED

C

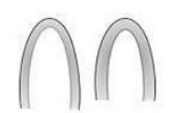

D

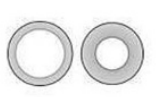

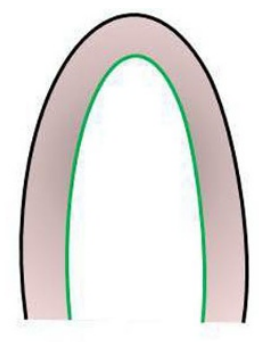

ES

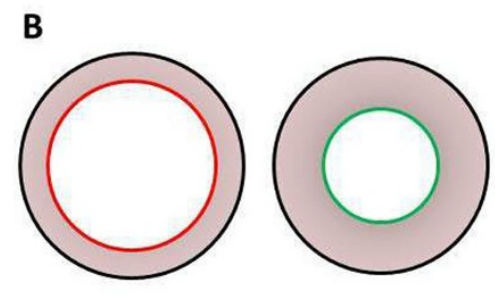

ES

Global Strain $\varepsilon[\%]=\frac{L_{E S}-L_{E D}}{L_{E D}} \times 100$

\section{Figure 1}

Published: 3 February 2015

doi:10.1186/1532-429X-17-S1-Q9

Cite this article as: Riffel et al:: Standardized assessment of global longitudinal and circumferential strain - a modality independent

software approach. Journal of Cardiovascular Magnetic Resonance 201517

(Suppl 1):Q9.

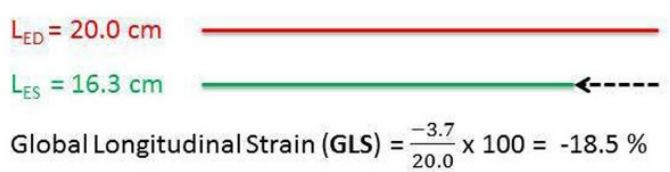

$\mathrm{L}_{\mathrm{ED}}=13.0 \mathrm{~cm}$

$L_{E S}=8.8 \mathrm{~cm}$

Global Circumferential Strain $($ GCS $)=\frac{-4.2}{13.0} \times 100=-32.3 \%$ 